\title{
The Value of Lateral Flow Urine Lipoarabinomannan Assay and Empirical Treatment in the Xpert MTB/RIF Ultra Era: a Prospective Cohort Study
}

Wakjira Kebede ( $\square$ wakjirakebede@yahoo.com )

Jimma University

Gemeda Abebe

Jimma University

Esayas Kebede Gudina

Jimma University

Annelies Van Rie

University of Antwerp

\section{Research Article}

Keywords: tuberculosis, molecular diagnostics, LF-LAM, Xpert MTB/RIF Ultra, rifampicin, empiric treatment, sputum, urine, Ethiopia

Posted Date: June 2nd, 2021

DOl: https://doi.org/10.21203/rs.3.rs-567885/v1

License: (9) (1) This work is licensed under a Creative Commons Attribution 4.0 International License.

Read Full License 


\section{Abstract}

Introduction: The value of Lateral Flow urine Lipoarabinomannan (LF-LAM) assay and the role of empiric tuberculosis (TB) treatment in the era of the highly sensitive Xpert MTB/RIF Ultra (Xpert Ultra) assay is unclear. This study aimed to assess the additional yield of Xpert Ultra compared to Xpert MTB/RIF, the yield of LF-LAM assay when used in combination with Xpert Ultra, and the role of empiric TB treatment in the Xpert Ultra era among patients with symptoms of TB admitted to a tertiary hospital in Ethiopia.

Methods: We performed a secondary analysis of a cohort of hospitalized Xpert MTB/RIF-negative patients. Sputum samples were examined for Mycobacterium tuberculosis by culture and Xpert Ultra. In HIV positive and severely ill patients, a urine sample was collected for the LF-LAM assay. Treatment outcome was assessed six months after enrollment. Logistic regression was used to identify factors predictive of deaths among Xpert Ultra negative patients.

Results: Of the 250 hospitalized Xpert MTB/RIF-negative patients, 35 (14.0\%) had culture-confirmed TB. The Xpert Ultra assay diagnosed 31 of the 35 culture positive cases that had been missed by Xpert MTB/RIF. The LF-LAM assay did not identify any case that was not detected by Xpert Ultra. Empiric treatment did not improve survival among Xpert Ultra negative patients (OR 1.28, 95\% Cl: 0.3-5.3). Low body mass index $\left(<18.5 \mathrm{~kg} / \mathrm{m}^{2}\right)$ was the only significant predictor of death in Xpert Ultra negative patients (OR 4.0, 95\% Cl: 1.12-16.5).

Conclusions: In this prospective cohort, we observed that LF-LAM did not improve the diagnostic yield when used in combination with Xpert Ultra and did not find a survival benefit of empiric treatment among Xpert Ultra negative individuals. Research is needed to determine the optimal management of patients with presumptive TB and a negative Xpert Ultra assay in high TB burden settings.

\section{Introduction}

Tuberculosis (TB) is a chronic infectious disease caused by Mycobacterium tuberculosis (MTB) complex [1]. With an estimated 10 million active TB cases, of which $10 \%$ are co-infected with HIV, and 1.3 million deaths in 2019, TB remains a public health problem worldwide [2]. In 2019, Ethiopia had the 10th highest incidence of TB globally, with 114, 233 new cases and an incidence rate of 151 cases per 100, 000 people $[2,3]$.

Even though the World Health Organization (WHO) endorsed the molecular Xpert MTB/RIF assay almost a decade ago [4], many primary care clinics in high burden countries still rely on smear microscopy as the initial diagnostic test. Because of the low sensitivity of smear microscopy, smear negative patients with TB symptoms are often empirically started on TB treatment [5]. Even when the Xpert MTB/RIF assay is available, empirical treatment remains highly prevalent in high TB burden countries [6]. The use of empirical treatment can be justified in these settings given the suboptimal performance of the Xpert assay, especially among smear negative individuals (sensitivity of $67 \%$ ) and in people living with HIV (sensitivity of $80 \%$ ) $[7,8]$. 
In 2017, the WHO recommended replacing Xpert MTB/RIF by Xpert MTB/RIF Ultra (Xpert Ultra) as the initial diagnostic test because of its superior performance in smear negative patients and patients living with HIV [9]. A meta-analysis of 19 studies found an overall pooled sensitivity of $84 \%$ the Xpert Ultra compared to $69 \%$ for Xpert MTB/RIF [10]. In smear-negative TB cases ,Xpert Ultra was $17 \%$ more sensitive compared to Xpert MTB/RIF; in people living with HIV Xpert Ultra was 13\% more sensitive [11-13]. Whether empiric TB treatment is still justified in high TB burden settings in the Xpert Ultra era has not yet been investigated.

The lateral flow urine lipoarabinomannan assay (LF-LAM) is another tuberculosis diagnostic tool that was recently endorsed but is not yet commonly used in high TB burden countries [14]. In inpatient settings, LF-LAM can assist in the diagnosis of TB in people living with HIV and in people who are seriously ill. Among people living with HIV who present with signs and symptoms of TB to an inpatient setting, the pooled sensitivity of LF-LAM was $52 \%(40-64 \%)$ and pooled specificity $87 \%$ (78-93\%). When used in combination with other tests, LF-LAM can improve the diagnostic yield by $14 \%$ (compared to Xpert MTB/RIF alone) or $36.6 \%$ (compared to clinical sign and symptoms alone) $[15,16]$. It has also been shown that the use of LF-LAM can improve the survival of patients hospitalized with advanced HIV disease [pooled risk ratio for mortality $0.85(0.76-0.94)][14,17,18]$. The yield of LAM when used in combination with Xpert Ultra has not yet been assessed.

In this study, we aimed to assess the additional yield of Xpert Ultra compared to Xpert MTB/RIF, the yield of LF-LAM assay when used in combination with Xpert Ultra, and the role of empiric TB treatment in the Xpert Ultra era among patients with symptoms of TB admitted to a tertiary hospital in Ethiopia.

\section{Materials And Methods}

\section{Study setting}

The study was conducted in Jimma University Medical Center, a tertiary hospital located in Jimma, Oromia Region, Ethiopia. Patients are referred to the Center from health centers, district hospitals and private health facilities in Jimma Zone and district hospitals in neighboring regions, resulting in a catchment population of over 20 million. The hospital has about 800 inpatient beds and about 20000 inpatients, 220000 outpatient, and 15000 emergency cases visit the hospital annually [19]. During the study period (December 2018 to July 2019), the Xpert MTB/RIF assay was used as the initial test for diagnosing TB in all people presenting with signs and symptoms of TB at the Jimma University Medical Center [20]. The Mycobacteriology Research Center of Jimma University, which is located in close proximity of the medical center, serves as the TB reference laboratory for southwest Ethiopia.

\section{Study population and data collection}

This analysis is a secondary analysis of a cohort study that aimed to determine the role of empiric treatment among hospitalized adults (age $\geq 18$ years) with symptoms of pulmonary TB (current cough, night sweats, fever, and weight loss) who tested negative on the Xpert MTB/RIF assay [21]. The cohort 
study enrolled 125 patients treated empirically for active TB and 125 patients in whom the physician decided not to treat for TB. A structured questionnaire was administered by trained nurses to collect demographic and clinical characteristic and medical records of the patients were reviewed to document HIV status, CD 4 count, and improvement of TB symptoms on TB treatment. Sputum samples were collected in the first 48 hours following enrolment for a single Xpert Ultra assay, liquid culture using the Mycobacteria Growth Indicator Tube (MGIT) system and solid culture on Lowenstein Jensen (LJ). Drug susceptibility testing for first line anti-TB drugs (rifampicin and isoniazid) was done by Line Probe assay (LPA). HIV-positive and severely ill patients (temperature $>39^{\circ} \mathrm{C}$, respiratory rate $>30 \mathrm{resp} . / \mathrm{min}$, cardiac rate $>120 \mathrm{bpm}$, or unable to walk without help) [22] were asked to provide a $10 \mathrm{ml}$ urine sample for a LF-LAM assay. All laboratory tests were performed at the Mycobacteriology Research Center of Jimma University.

\section{Laboratory procedures}

\section{Mycobacterial culture}

Sputum sample decontamination and digestion, inoculation of concentrated sputum samples on LJ and MGIT media were performed using standard procedures [23]. The mycobacterial culture was classified as positive when growth was detected by LJ and/or MGIT with a positive SD Bioline TB antigen MPT64 confirmatory test and a negative blood agar test. Culture negative denotes the absence of growth on both LJ and MGIT culture or positive LJ/MGIT culture with a negative SD Bioline TB antigen MPT64 result.

\section{Xpert MTB/RIF Ultra test}

Xpert Ultra test was performed according to manufacturer's instruction [24]. Briefly, $1 \mathrm{ml}$ of sputum was added to $2 \mathrm{ml}$ of reagent in a 15-ml Falcon tube, vortexed for at least 10 seconds and incubated at room temperature for 10 minutes. The mixture was then added to the Xpert Ultra assay cartridge and placed in the instrument. The Xpert Ultra test was repeated using the same sample in cases of an invalid result. In cases of "trace" result, a new sputum sample was collected for a second Xpert Ultra test. If the second test was positive (trace or higher), the result was considered positive for patients living with HIV and for HIV-negative patients without a history of TB treatment.

\section{LF-LAM test}

The urine Determine TB-LAM test (Abbott Laboratories, Lake Bluff, USA; formerly Alere Inc, Waltham, USA) was performed by applying $60 \mu \mathrm{L}$ of fresh urine to the sample pad at the bottom of the test strip using a micro-pipette. After 25 minutes of incubation at room temperature, the test strip was inspected visually. If any band is observed, the intensity was scored as grade 1, 2, 3 or 4 as compared with the reference card. The test was read independently by two certified laboratory professionals in blinded manner (blinded to each other and the sputum Xpert Ultra and culture results). LAM test results were classified as positive if grade $\geq 1$ and as negative if no test band was observed [16].

\section{Data analysis}


Descriptive statistics were performed using frequencies and percentages to describe the characteristics of the study population. The yield of Xpert Ultra and LAM tests was determined by comparison to mycobacterial culture results. Mortality in the 6 months post assessment was assessed among Xpert Ultra negative participants, stratified by empiric treatment status. To identify factors associated with mortality among Xpert Ultra negative patients, we calculated the odds ration using a logistic regression model. Data analysis was performed using R Statistical software version 3.6.1.

\section{Ethical consideration}

Ethical clearance was obtained from the Ethical Review Board of Institute of Health of Jimma University (Ref. no: IHRPGD/397/2018). Written informed consent was obtained from all study participants. All methods were performed in accordance with the relevant guidelines and regulations. Clinicians treating study participants in whom TB was bacteriologically confirmed were notified of the result.

\section{Results}

\section{Characteristics of the study population}

All 250 patients admitted to the hospital with signs or symptoms of TB who were enrolled in the parent study were included in the secondary analysis. By design, all study participants had a negative Xpert MTB/RIF result; 125 were empirically initiated on TB treatment by the treating physician and the physician did not start TB treatment in the other 125 participants (Fig. 1). The majority were 40 years of age or younger (144/250,57.6\%), female (138/250,55.2\%), and rural residents (154/250, 57.6\%). Almost all patients $(232 / 250,92.8 \%)$ had already visited another health facilities for similar complaints before their current hospitalization. HIV status was documented for 243 (97.2\%) participants of which $21.4 \%$ were positive (Table 1). All participants had symptoms of current cough, fever, night sweat and shortness of breath; $165(66 \%)$ had a cough for $\geq 14$ days, 45 (18\%) had a history of previous TB treatment and $(93 / 250,37.6 \%)$ had a chest X-ray findings typical or compatible with TB. 
Table 1

Demographic and clinical characteristics of 250 Xpert MTB/RIF-negative patients hospitalized at Jimma University Medical Center, Oromia, Ethiopia.

\begin{tabular}{|c|c|c|c|c|}
\hline \multirow[t]{2}{*}{ Characteristics } & \multirow[t]{2}{*}{ Category } & \multirow[t]{2}{*}{ All } & \multicolumn{2}{|c|}{$\begin{array}{l}\text { Xpert Ultra test } \\
\text { results }\end{array}$} \\
\hline & & & Positive & Negative \\
\hline Patients n & & 250 & 35 & 215 \\
\hline \multirow[t]{2}{*}{ Age } & $\leq 40$ years & $\begin{array}{l}144 \\
(57.6)\end{array}$ & $\begin{array}{l}29 \\
(82.9)\end{array}$ & $\begin{array}{l}115 \\
(53.5)\end{array}$ \\
\hline & $>40$ years & $\begin{array}{l}106 \\
(42.4)\end{array}$ & $6(17.1)$ & $\begin{array}{l}100 \\
(46.5)\end{array}$ \\
\hline \multirow[t]{2}{*}{ Sex } & Female & $\begin{array}{l}138 \\
(55.2)\end{array}$ & $\begin{array}{l}22 \\
(62.9)\end{array}$ & $\begin{array}{l}116 \\
(54.0))\end{array}$ \\
\hline & Male & $\begin{array}{l}112 \\
(44.8)\end{array}$ & $\begin{array}{l}13 \\
(37.1)\end{array}$ & $\begin{array}{l}99 \\
(46.0)\end{array}$ \\
\hline \multirow[t]{3}{*}{ Educational status } & Illiterate & $\begin{array}{l}116 \\
(46.4)\end{array}$ & $8(22.9)$ & $\begin{array}{l}108 \\
(50.2)\end{array}$ \\
\hline & Primary level & $\begin{array}{l}51 \\
(20.4)\end{array}$ & $8(22.9)$ & $\begin{array}{l}43 \\
(20.0)\end{array}$ \\
\hline & Secondary \& above & $\begin{array}{l}83 \\
(33.2)\end{array}$ & $\begin{array}{l}19 \\
(54.2)\end{array}$ & $\begin{array}{l}64 \\
(29.8)\end{array}$ \\
\hline \multirow[t]{2}{*}{ Residence } & Urban & $\begin{array}{l}106 \\
(42.4)\end{array}$ & $\begin{array}{l}15 \\
(42.9)\end{array}$ & $\begin{array}{l}91 \\
(42.3)\end{array}$ \\
\hline & Rural & $\begin{array}{l}144 \\
(57.6)\end{array}$ & $\begin{array}{l}20 \\
(57.1)\end{array}$ & $\begin{array}{l}124 \\
(57.7)\end{array}$ \\
\hline \multirow[t]{4}{*}{$\begin{array}{l}\text { Health facilities visited prior to hospital } \\
\text { admission }\end{array}$} & $\begin{array}{l}\text { Local government } \\
\text { hospitals }\end{array}$ & $\begin{array}{l}126 \\
(50.4)\end{array}$ & $9(25.7)$ & $\begin{array}{l}117 \\
(54.4)\end{array}$ \\
\hline & Primary care center & $\begin{array}{l}48 \\
(19.2)\end{array}$ & $\begin{array}{l}10 \\
(28.6)\end{array}$ & $\begin{array}{l}38 \\
(17.7)\end{array}$ \\
\hline & Private Clinic & $\begin{array}{l}58 \\
(23.2)\end{array}$ & $\begin{array}{l}13 \\
(37.1)\end{array}$ & $\begin{array}{l}45 \\
(20.9)\end{array}$ \\
\hline & None & $18(7.2)$ & $3(8.6)$ & $15(7.0)$ \\
\hline \multirow[t]{2}{*}{ Body mass index } & $\leq 18.5 \mathrm{~kg} / \mathrm{m} 2$ & $\begin{array}{l}84 \\
(33.6)\end{array}$ & $\begin{array}{l}22 \\
(62.9)\end{array}$ & $\begin{array}{l}62 \\
(28.8)\end{array}$ \\
\hline & $>18.5 \mathrm{~kg} / \mathrm{m} 2$ & $\begin{array}{l}166 \\
(66.4)\end{array}$ & $\begin{array}{l}13 \\
(37.1)\end{array}$ & $\begin{array}{l}153 \\
(71.2)\end{array}$ \\
\hline
\end{tabular}

Data are presented as $\mathrm{n}(\%)$, unless otherwise stated. 


\begin{tabular}{|c|c|c|c|c|}
\hline \multirow{2}{*}{$\begin{array}{l}\text { Characteristics } \\
\text { HIV status }\end{array}$} & \multirow{2}{*}{$\begin{array}{l}\text { Category } \\
\text { Negative }\end{array}$} & \multirow{2}{*}{$\begin{array}{l}\text { All } \\
\begin{array}{l}191 \\
(76.4)\end{array}\end{array}$} & \multicolumn{2}{|c|}{$\begin{array}{l}\text { Xpert Ultra test } \\
\text { results }\end{array}$} \\
\hline & & & $\begin{array}{l}24 \\
(68.8)\end{array}$ & $\begin{array}{l}167 \\
(77.7)\end{array}$ \\
\hline & Positive & $\begin{array}{l}52 \\
(20.8)\end{array}$ & $\begin{array}{l}11 \\
(31.4)\end{array}$ & $\begin{array}{l}41 \\
(19.1)\end{array}$ \\
\hline & Unknown & $7(2.8)$ & $0(0.0)$ & $7(3.2)$ \\
\hline \multirow[t]{2}{*}{ CD4 count $(n=52)$} & $\leq 200 \mathrm{cells} / \mathrm{ml}$ & $\begin{array}{l}24 \\
(46.2)\end{array}$ & $\begin{array}{l}10 \\
(90.9)\end{array}$ & $\begin{array}{l}14 \\
(26.9)\end{array}$ \\
\hline & $>200$ cells/ml & $\begin{array}{l}28 \\
(53.8)\end{array}$ & $1(9.1)$ & $\begin{array}{l}27 \\
(73.1)\end{array}$ \\
\hline \multirow[t]{2}{*}{ History of TB treatment } & No & $\begin{array}{l}205 \\
(82.0)\end{array}$ & $\begin{array}{l}27 \\
(77.1)\end{array}$ & $\begin{array}{l}178 \\
(82.8)\end{array}$ \\
\hline & Yes & $\begin{array}{l}45 \\
(18.0)\end{array}$ & $8(22.9)$ & $\begin{array}{l}37 \\
(17.2)\end{array}$ \\
\hline \multirow[t]{2}{*}{ Clinical severity } & Non-severe & $\begin{array}{l}232 \\
(92.8)\end{array}$ & $\begin{array}{l}30 \\
(85.7)\end{array}$ & $\begin{array}{l}202 \\
(94.0)\end{array}$ \\
\hline & Severe & $18(7.2)$ & $5(13.9)$ & $13(6.0)$ \\
\hline \multirow[t]{2}{*}{ Weight loss } & No & $\begin{array}{l}111 \\
(44.4)\end{array}$ & $9(25.7)$ & $\begin{array}{l}102 \\
(47.4)\end{array}$ \\
\hline & Yes & $\begin{array}{l}139 \\
(55.6)\end{array}$ & $\begin{array}{l}26 \\
(74.3)\end{array}$ & $\begin{array}{l}113 \\
(52.6)\end{array}$ \\
\hline \multirow[t]{2}{*}{ Pleuritic chest pain } & No & $\begin{array}{l}82 \\
(32.8)\end{array}$ & $5(14.3)$ & $\begin{array}{l}77 \\
(35.8)\end{array}$ \\
\hline & Yes & $\begin{array}{l}168 \\
(67.2)\end{array}$ & $\begin{array}{l}30 \\
(85.7)\end{array}$ & $\begin{array}{l}138 \\
(64.2)\end{array}$ \\
\hline \multirow[t]{2}{*}{ Loss of appetite } & No & $\begin{array}{l}49 \\
(19.6)\end{array}$ & $2(5.7)$ & $\begin{array}{l}47 \\
(21.9)\end{array}$ \\
\hline & Yes & $\begin{array}{l}201 \\
(80.4)\end{array}$ & $\begin{array}{l}33 \\
(94.3)\end{array}$ & $\begin{array}{l}168 \\
(78.1)\end{array}$ \\
\hline \multirow[t]{2}{*}{ Duration of cough } & $<14$ days & $\begin{array}{l}85 \\
(34.0)\end{array}$ & $5(14.3)$ & $\begin{array}{l}80 \\
(37.2)\end{array}$ \\
\hline & $\geq 14$ days & $\begin{array}{l}165 \\
(66.0)\end{array}$ & $\begin{array}{l}30 \\
(85.7)\end{array}$ & $\begin{array}{l}135 \\
(62.8)\end{array}$ \\
\hline Duration of fever & $<14$ days & $\begin{array}{l}137 \\
(54.8)\end{array}$ & $\begin{array}{l}17 \\
(48.6)\end{array}$ & $\begin{array}{l}120 \\
(55.8)\end{array}$ \\
\hline
\end{tabular}

Data are presented as n (\%), unless otherwise stated. 


\begin{tabular}{|lllll|}
\hline Characteristics & Category & All & \multicolumn{2}{l|}{$\begin{array}{l}\text { Xpert Ultra test } \\
\text { results }\end{array}$} \\
\hline & $\geq 14$ days & $\begin{array}{l}113 \\
(45.2)\end{array}$ & $\begin{array}{l}18 \\
(51.4)\end{array}$ & $\begin{array}{l}95 \\
(44.2)\end{array}$ \\
\hline Duration of night sweats & $<14$ days & 130 & 18 & 112 \\
& & $(52.0)$ & $(51.4)$ & $(52.1)$ \\
\cline { 2 - 5 } & $\geq 14$ days & 120 & 17 & 103 \\
& & $(48.0)$ & $(48.6)$ & $(47.9)$ \\
\hline Duration of shortness of breath & $<14$ days & 123 & 10 & 113 \\
& & $(49.2)$ & $(28.6)$ & $(52.6)$ \\
\cline { 2 - 5 } & $\geq 14$ days & 127 & 25 & 102 \\
& & $(50.8)$ & $(71.4)$ & $(47.4)$ \\
\hline Data are presented as $n$ (\%), unless otherwise stated. & & & \\
\hline
\end{tabular}

\section{Results of diagnostic tests}

Of the 250 cultures, 35 (14.0\%) were positive for MTB complex and $2(0.8 \%)$ for non-tuberculosis mycobacteria (NTM), 202/250 (80.8\%) were MTB culture negative and 11/250 (4.4\%) were contaminated (Table 2). 
Table 2

Xpert Ultra and LAM test results compared with mycobacterial culture for the diagnosis of pulmonary TB among Xpert MTB/RIF-negative patients hospitalized at Jimma University Medical Center, Oromia,

\section{Ethiopia.}

\begin{tabular}{|c|c|c|c|c|c|}
\hline & \multicolumn{5}{|c|}{ Mycobacterial culture results } \\
\hline & $\begin{array}{l}\text { LJ or MGIT } \\
\text { positive }\end{array}$ & $\begin{array}{l}\text { LJ or MGIT } \\
\text { negative }\end{array}$ & $\begin{array}{l}\text { LJ or MGIT } \\
\text { contaminated }\end{array}$ & NTM & Total \\
\hline \multicolumn{6}{|l|}{$\begin{array}{l}\text { Xpert MTB/RIF } \\
\text { Ultra }\end{array}$} \\
\hline Mtb detected* & $27(77.2)$ & $0(0.0)$ & $4(36.4)$ & $\begin{array}{l}0 \\
(0.0)\end{array}$ & $\begin{array}{l}31 \\
(12.4)\end{array}$ \\
\hline Mtb Trace & $4(11.4)$ & $0(0.0)$ & $0(0.0)$ & $\begin{array}{l}0 \\
(0.0)\end{array}$ & $4(1.6)$ \\
\hline $\begin{array}{l}\text { Mtb not } \\
\text { detected }\end{array}$ & $4(11.4)$ & $202(100)$ & $7(63.6)$ & 2 & $\begin{array}{l}211 \\
(84.4)\end{array}$ \\
\hline Total & 35 & 202 & 11 & 2 & 250 \\
\hline \multicolumn{6}{|l|}{$\begin{array}{l}\text { LF-LAM }(n= \\
\left.68^{* *}\right)\end{array}$} \\
\hline Positive & $7(58.3)$ & $2(3.8)$ & $0(0.0)$ & $\begin{array}{l}0 \\
(0.0)\end{array}$ & $9(13.2)$ \\
\hline Negative & $4(41.7)$ & $52(96.2)$ & $2(100)$ & $\begin{array}{l}1 \\
(100)\end{array}$ & $\begin{array}{l}59 \\
(86.8)\end{array}$ \\
\hline Total & 11 & 54 & 2 & 1 & 68 \\
\hline \multicolumn{6}{|c|}{ Data are presented as $\mathrm{n}(\%)$, unless otherwise stated. } \\
\hline \multicolumn{6}{|c|}{$\begin{array}{l}\text { Abbreviations: Mtb, Mycobacterium tuberculosis; NTM, Non tuberculosis mycobacteria; LJ, } \\
\text { Lowenstein Jensen medium; MGIT, Mycobacteria Growth Indicator Tube; TB, Tuberculosis; LF-LAM, } \\
\text { Lateral Flow urine Lipoarabinomannan. }\end{array}$} \\
\hline \multicolumn{6}{|c|}{ *All were sensitive to rifampicin. } \\
\hline $\begin{array}{l}\text { ** Urine TB-LAN } \\
\text { HIV-negative pa }\end{array}$ & $\begin{array}{l}\text { was perfor } \\
\text { ants }(2 \mathrm{hac}\end{array}$ & $\begin{array}{l}\text { all } 52 \text { HIV-p } \\
\text { amples mis }\end{array}$ & rticipants anc & $18 \mathrm{se}$ & dy ill \\
\hline
\end{tabular}

The Xpert Ultra assay was positive for MTB complex in 35/250 (14\%), of which 4 (11.4\%) had a trace call. The Xpert Ultra result was negative in 215 (86.0\%) participants, including four samples that were repeated because of an error result on the initial test (Table 2). The Xpert Ultra assay was positive in 4 patients with a contaminated culture. One rifampicin resistant strain identified by LPA was not identified as rifampicin resistant by Xpert Ultra. 
All 52 HIV-positive and 16 of the 18 severely ill HIV-negative participants provided a urine sample, of which $9(13.2 \%)$ had a positive LF-LAM result (Fig. 2). The inter-reader agreement was $97.1 \%$ (kappa = 0.87).

\section{Yield of Xpert Ultra and role of LF-LAM tests}

Participants whose sputum cultures were contaminated $(n=11)$ or positive for NTM $(n=2)$ were excluded from this analysis. Of the remaining 237 participants, 35 (14.8\%) had culture-confirmed TB. The Xpert Ultra assay detected $31(88.6 \%)$ of the 35 culture-confirmed cases. The 4 culture-confirmed cases that were missed by Xpert Ultra were HIV negative. The assay was positive in $4(2.0 \%)$ and was negative in 198 (98.0\%) of the 202 culture-negative participants. The 4 Xpert Ultra positive culture negative cases had very low mycobacterial burden as demonstrated by a high cycle threshold (Ct) value (Ct value of the lowest probe $\geq 29.2$, Supplemental Table), were all HIV negative, and none of these cases had a history of prior TB treatment.

The 68 people living with HIV or seriously ill individual assessed by LF-LAM, one participant was excluded from the analysis because of presence of NTM $(n=1)$. Among the remaining 67 participants, nine LFLAM tests were positive. Seven of the 11 (63.6\%) culture positive cases were detected by LF-LAM. These seven cases were also were positive Xpert Ultra. In addition, two positive LF-LAM assays occurred in four of the Xpert Ultra positive but culture negative participants (Fig. 2). The LF-LAM assay thus did not increase the diagnostic yield when used in combination with Xpert Ultra.

\section{Role of empiric TB treatment in the Xpert Ultra era}

Of the 215 (86\%) Xpert Ultra negative participants, 94 (43.7\%) were diagnosed clinically and empirically started on TB treatment, and 121 (56.3\%) were clinically considered not to have TB and were not initiated on TB treatment. Of the 94 Xpert Ultra negative participants started on empiric TB treatment, 90 (95.7\%) were alive and $4(4.3 \%)$ had died in the 6 months following assessment. Of the 121 Xpert Ultra negative patients not started on TB treatment, 115 (95.0\%) were alive and 6 (5.0\%) had died in the 6 months following assessment (Table 3). Among Xpert Ultra negative patients, the odds of survival was similar between those who did and did not receive empiric TB treatment (OR 1.28, 95\% $\mathrm{Cl} 0.3-5.3$ ). 
Table 3

Role of empiric TB treatment in the Xpert Ultra era among Xpert MTB/RIF-presumptive TB cases hospitalized at Jimma University Medical Center, Oromia, Ethiopia.

\begin{tabular}{|c|c|c|c|}
\hline \multirow[t]{2}{*}{ TB treatment status \& outcomes } & \multirow[t]{2}{*}{ All patients } & \multicolumn{2}{|c|}{ Xpert Ultra test results } \\
\hline & & Positive & Negative \\
\hline Empirically initiated on TB treatment & $125 / 250(50.0)$ & $31 / 35(86.6)$ & $94 / 215(43.7)$ \\
\hline \multicolumn{4}{|l|}{ Outcome } \\
\hline alive & $118 / 125(94.4)$ & 28/31 (90.3) & $90 / 94(95.7)$ \\
\hline died & $7 / 125(5.6)$ & $3 / 31(9.7)$ & $4 / 94(4.3)$ \\
\hline Not initiated on TB treatment & $125 / 250(50.0)$ & 4/35 (11.4) & $121 / 215(56.3)$ \\
\hline \multicolumn{4}{|l|}{ Outcome } \\
\hline alive & $119 / 125$ (95.2) & $\#_{4 / 4}(100.0)$ & $115 / 121(95.0)$ \\
\hline died & $6 / 125(4.8)$ & $0 / 4(0.0)$ & $6 / 121(5.0)$ \\
\hline Total & 250 & 35 & 215 \\
\hline \multicolumn{4}{|c|}{ Data are presented as $n(\%)$, unless otherwise stated. } \\
\hline
\end{tabular}

\section{Predictor of 6-month mortality among Xpert Ultra negative patients}

In regression analysis, it was found that underweight or body mass index $(\mathrm{BMI})<18.5 \mathrm{~kg} / \mathrm{m} 2$ was significant predictor of mortality among Xpert Ultra negative patients (OR 4.0, 95\% Cl: 1.12-16.5) (Table 4). 
Table 4

Patient characteristics associated with mortality among Xpert Ultra negative patients hospitalized at Jimma University Medical Center, Oromia, Ethiopia.

\begin{tabular}{|c|c|c|c|c|}
\hline \multirow[t]{2}{*}{ Characteristics } & \multirow[t]{2}{*}{ Category } & \multicolumn{2}{|c|}{ Outcome } & \multirow[t]{2}{*}{ Odds Ratio (95\% Cl) } \\
\hline & & Died & Alive & \\
\hline Patients n & & 10 & 205 & \\
\hline \multirow[t]{2}{*}{ Age } & $\leq 40$ years & $5(50.0)$ & $108(52.7)$ & Ref. \\
\hline & $>40$ years & $5(50.0)$ & $97(47.3)$ & $1.11(0.3-4.1)$ \\
\hline \multirow[t]{2}{*}{ Sex } & Female & $5(50.0)$ & $112(54.6)$ & Ref. \\
\hline & Male & $5(50.0)$ & $93(45.4)$ & $1.2(0.3-4.4)$ \\
\hline \multirow[t]{3}{*}{ Educational status } & $\begin{array}{l}\text { Secondary \& } \\
\text { above }\end{array}$ & $1(10.0)$ & $43(21.0)$ & Ref. \\
\hline & Primary level & $3(30.0)$ & $60(29.3)$ & $2.1(0.26-44.2)$ \\
\hline & Illiterate & $6(60.0)$ & $102(49.8)$ & $2.5(0.41-48.5)$ \\
\hline \multirow[t]{2}{*}{ Residence } & Urban & $5(50.0)$ & $87(42.4)$ & Ref. \\
\hline & Rural & $5(50.0)$ & $118(57.7)$ & $0.73(0.2-2.7)$ \\
\hline \multirow[t]{2}{*}{ Clinical severity } & Non-severe & $9(90.0)$ & $190(94.1)$ & Ref. \\
\hline & Severe & $1(10.0)$ & $12(5.9)$ & $1.7(0.19-10.7)$ \\
\hline \multirow[t]{2}{*}{ Body mass index } & $>18.5 \mathrm{Kg} / \mathrm{m}^{2}$ & $4(40.0)$ & $150(73.2)$ & Ref. \\
\hline & $\leq 18.5 \mathrm{Kg} / \mathrm{m}^{2}$ & $6(60.0)$ & $55(26.8)$ & $4.0(1.12-16.5)^{*}$ \\
\hline \multirow[t]{2}{*}{ HIV status ${ }^{9}$} & Negative & $6(60.0)$ & $160(78.0)$ & Ref. \\
\hline & Positive & $4(40.0)$ & 38 (18.5) & $2.8(0.7-10.3)$ \\
\hline \multirow[t]{2}{*}{ Weight loss } & No & $4(40.0)$ & $99(48.3)$ & Ref. \\
\hline & Yes & $6(60.0)$ & $106(51.7)$ & $1.4(0.38-5.61)$ \\
\hline \multirow[t]{2}{*}{ Loss of appetite } & No & $1(10.0)$ & $46(22.4)$ & Ref. \\
\hline & Yes & $9(90.0)$ & $159(77.6)$ & $2.6(0.47-48.6)$ \\
\hline \multirow[t]{2}{*}{ Pleuritic chest pain } & No & $4(40.0)$ & $74(36.1)$ & Ref. \\
\hline & yes & $6(60.0)$ & $131(63.9)$ & $0.84(0.23-3.4)$ \\
\hline
\end{tabular}

Data are presented as $\mathrm{n}(\%),{ }^{\star} \mathrm{p}$ value 0.037 ; $\mathrm{Cl}$, confidence interval; ${ }^{\uparrow} 7$ missing HIV test results 


\begin{tabular}{|c|c|c|c|c|}
\hline \multirow[t]{2}{*}{ Characteristics } & \multirow[t]{2}{*}{ Category } & \multicolumn{2}{|c|}{ Outcome } & \multirow[t]{2}{*}{ Odds Ratio (95\% Cl) } \\
\hline & & Died & Alive & \\
\hline \multirow[t]{2}{*}{ History of TB treatment } & No & $9(90.0)$ & $169(82.4)$ & Ref. \\
\hline & Yes & $1(10.0)$ & $36(17.6)$ & $0.5(0.02-2.9)$ \\
\hline \multirow[t]{2}{*}{ Duration of fever } & $<14$ days & $6(60.0)$ & $115(56.1)$ & Ref. \\
\hline & $\geq 14$ days & $4(40.0)$ & $90(43.9)$ & $0.85(0.21-3.07)$ \\
\hline \multirow[t]{2}{*}{ Duration of cough } & $<14$ days & $4(40.0)$ & $77(37.6)$ & Ref. \\
\hline & $\geq 14$ days & $6(60.0)$ & $128(62.4)$ & $0.9(0.24-3.62)$ \\
\hline \multirow[t]{2}{*}{ Duration of night sweats } & $<14$ days & $6(60.0)$ & $107(52.2)$ & Ref. \\
\hline & $\geq 14$ days & $4(40.0)$ & $98(47.8)$ & $0.72(0.18-2.62)$ \\
\hline \multirow{2}{*}{$\begin{array}{l}\text { Duration of shortness of } \\
\text { breath }\end{array}$} & $<14$ days & $5(50.0)$ & $109(53.2)$ & Ref. \\
\hline & $\geq 14$ days & $5(50.0)$ & $96(46.8)$ & $1.13(0.30-4.19)$ \\
\hline \multirow[t]{2}{*}{ Culture results } & Negative & $9(90.0)$ & $202(98.5)$ & Ref. \\
\hline & Positive & $1(10.0)$ & $3(1.5)$ & $7.48(0.35-65.5)$ \\
\hline \multirow[t]{2}{*}{ Empiric treatment } & No & $6(60.0)$ & $115(56.1)$ & Ref. \\
\hline & Yes & $4(40.0)$ & $90(43.9)$ & $0.85(0.21-3.07)$ \\
\hline
\end{tabular}

\section{Discussion}

In this prospective cohort study, we confirmed the high sensitivity of Xpert Ultra among cases of presumptive TB, as $89 \%$ (31 of the 35 ) of the culture positive cases missed by Xpert MTB/RIF were positive on Xpert Ultra. We further demonstrated that the use of empirical treatment may no longer be justified in the Xpert Ultra era and that the recommendation for use of a LF-LAM may need to be revised for settings where access to Xpert Ultra is available.

These observed high sensitivity of Xpert Ultra is in line with a meta-analyses, where $87.2 \%$ of TB culture positive patients were positive on Xpert Ultra test [12] and an overall estimated pooled sensitivity of $90.9 \%$ (95\% credible interval 86.2 to 94.7 ) [25]. Similar to other studies, we also observed potentially false positive Xpert Ultra results, as 4 cases were Xpert Ultra positive by culture negative. False positive Xpert Ultra results have been associated with a recent history of TB treatment [11]. In our study, the Xpert Ultra positive culture negative cases had no history of previous TB treatment. Interestingly, two of the four cases had a positive LF-LAM result, suggesting that these cases may actually be true positive instead of false positive cases of TB. 
The LF-LAM assay detected $63.6 \%$ of the culture positive cases among people living with HIV or seriously ill presumptive TB cases. This corresponds to findings of a study performed in four countries in subSaharan Africa where a $60.0 \%$ sensitivity of LF-LAM was observed among culture positive cases [26]. In contrast to studies performed during the Xpert MTB/RIF era, where addition of LF-LAM to Xpert MTB/RIF assay increased the yield, the LF-LAM assay did not improve diagnostic yield as all LF-LAM positive patients were also positive on Xpert Ultra. These suggesting a limited role for LF-LAM in the Xpert Ultra era and, if confirmed in a future, larger study, may require revision of the guidelines for use of LF-LAM. In hospital settings where a Xpert Ultra assay is available, all LF-LAM negative cases would have to be reassessed by Xpert Ultra due to poor LAM sensitivity, and all LF-LAM positive cases would also need to reassessed by Xpert Ultra to determine whether there is resistance to rifampicin.

In high TB burden settings, treatment decisions often continue to rely on clinician's judgement and chest radiography findings, even when it has been shown that empiric treatment does not result in survival benefit $[6,27]$. In this study, we found that the Xpert Ultra assay detected almost all culture positive cases, questioning the value of reliance on empiric TB treatment for patients with negative Xpert Ultra results. Furthermore, we found that empiric treatment of Xpert Ultra patients did not increase the odds of survival, as survival was similar between those who did and did not receive empiric TB treatment (OR $1.28,95 \% \mathrm{Cl}$ : 0.3-5.3). These results suggest that the recommendation by Kendall et al and by Decroo et al, that clinicians should continue to prescribe TB treatment for Xpert MTB/RIF-negative patients whose clinical presentations strongly suggest pulmonary in order to minimize a risk of TB related mortality $[6,7]$ may no longer hold in the Xpert Ultra era. All participants in whom the clinician started empiric TB treatment had been assessed by chest radiography and had received a 'trial' of antibiotics (ceftriaxone and azithromycin, amoxicillin or vancomycin and doxycycline) to which they had not responded favorably. Given that empiric TB treatment in these Xpert Ultra negative patients did not improve survival, our results suggest that prescribing empiric treatment should be re-assessed as this may pose an unnecessary burden the health care system and may expose patients to unnecessary treatment. Future research should investigate which alternative diagnoses should be considered in these patient in order to develop evidence-based guidelines for the management of Xpert Ultra negative patients.

Our study had some limitations. First, we limited enrollment to hospitalized Xpert-negative patients, which limits generalizability of the outpatient settings. Second, our study was not powered to assess factors associated with mortality among people with presumptive TB who had a negative Xpert Ultra assay. Lastly, we did not assess the presence of other respiratory pathogens to explore the cause of symptoms or death in the Xpert Ultra negative patients.

In conclusion, Xpert Ultra assay provides a sensitive, specific and rapid diagnosis of TB among presumptive pulmonary TB cases. The use of LF-LAM test did not yield additional cases in Xpert Ultra negative patients. The odds of survival was similar between Xpert Ultra negative patients who did and did not receive empiric TB treatment. Our findings question the value of empiric treatment and the LF-LAM assay in the Xpert Ultra era. Future studies should be performed to provide the evidence needed to establish guidelines for the management of Xpert Ultra negative patients.

Page $14 / 19$ 


\section{Declarations}

\section{Acknowledgments}

The authors would like to thank the staff of the JUMC for their contribution to data collection and laboratory testing, Jimma University Mycobacteriology Research Center staffs for their technical assistance and the participants who participated in this study.

\section{Author contributions}

W.K., G.A. and A.V.R. designed the study. W.K. performed the laboratory test. G.A. and E.K.G. coordinated the study. W.K. and A.V.R. analyzed the data. W.K. wrote the first draft. All authors reviewed the manuscript.

\section{Funding}

This work was supported by the VLIR-UOS network project between Jimma University and a consortium of Flemish Universities, Belgium. The funder had no role in study design, data collection and analysis, decision to publish, or preparation of the manuscript.

\section{Competing interests}

The authors declare no competing interests.

\section{References}

1. Varaine F, Kenkens M, Grouzard V. Tuberculosis: Practical Guide to Clinicians, Nurses, Laboratory Technicians and Medical Auxiliaries. 5th ed ed. France, Paris: Medecins Sans Frontieres; 2010.

2. World health organization. Global tuberculosis report 2019. Geneva: World Health Organization, 2019 Contract No.: CC BY-NC-SA 3.0 IGO.

3. Neil AM, Glaziou P, Sismanidis C, Date A, Maloney S, Floyd K. Global Epidemiology of Tuberculosis and Progress Toward Meeting Global Targets-Worldwide, 2018. MMWR Morb Mortal Wkly Rep. 2020;69.

4. World health organization. Rapid implementation of the Xpert MTB/RIF diagnostic test: technical and operational „How-to®; practical considerations. Geneva, Switzerland: WHO; 2011.

5. Nakiyingi L, Bwanika JM, Kirenga B, Nakanjako D, Katabira C, Lubega G, et al. Clinical predictors and accuracy of empiric tuberculosis treatment among sputum smear-negative HIV-infected adult TB suspects in Uganda. PloS one. 2013;8(9):e74023. Epub 2013/09/17. doi:

10.1371/journal.pone.0074023.

6. Kendall EA, Kamoga C, Kitonsa PJ, Nalutaaya A, Salvatore PP, Robsky K, et al. Empiric treatment of pulmonary TB in the Xpert era: Correspondence of sputum culture, Xpert MTB/RIF, and clinical diagnoses. PloS one. 2019;14(7):e0220251. Epub 2019/07/25. doi: 10.1371/journal.pone.0220251. 
7. Decroo T, Henríquez-Trujillo AR, De Weggheleire A, Lynen L. Rational use of Xpert testing in patients with presumptive TB: clinicians should be encouraged to use the test-treat threshold. BMC infectious diseases. 2017;17(1):674. Epub 2017/10/13. doi: 10.1186/s12879-017-2798-6.

8. Hermans SM, Babirye JA, Mbabazi O, Kakooza F, Colebunders R, Castelnuovo B, et al. Treatment decisions and mortality in HIV-positive presumptive smear-negative TB in the Xpert ${ }^{\mathrm{TM}} \mathrm{MTB} / \mathrm{RIF}$ era: a cohort study. BMC infectious diseases. 2017;17(1):433. Epub 2017/06/18. doi: 10.1186/s12879-0172534-2.

9. World health organization. Next-generation Xpert® MTB/RIF Ultra assay recommended by WHO. Geneva, Swezerland: WHO; 2017.

10. Jiang J, Yang J, Shi Y, Jin Y, Tang S, Zhang N, et al. Head-to-head comparison of the diagnostic accuracy of Xpert MTB/RIF and Xpert MTB/RIF Ultra for tuberculosis: a meta-analysis. Infectious diseases (London, England). 2020;52(11):763-75. Epub 2020/07/04. doi:

$10.1080 / 23744235.2020 .1788222$.

11. Dorman SE, Schumacher SG, Alland D, Nabeta P, Armstrong DT, King B, et al. Xpert MTB/RIF Ultra for detection of Mycobacterium tuberculosis and rifampicin resistance: a prospective multicentre diagnostic accuracy study. The Lancet Infectious diseases. 2018;18(1):76-84. Epub 2017/12/05. doi: 10.1016/s1473-3099(17)30691-6.

12. Zhang $M$, Xue $M$, He JQ. Diagnostic accuracy of the new Xpert MTB/RIF Ultra for tuberculosis disease: A preliminary systematic review and meta-analysis. International journal of infectious diseases : IJID : official publication of the International Society for Infectious Diseases. 2020;90:3545. Epub 2019/09/24. doi: 10.1016/j.ijid.2019.09.016.

13. Kolia-Diafouka P, Carrère-Kremer $S$, Lounnas M, Bourdin A, Kremer L, Van de Perre P, et al. Detection of Mycobacterium tuberculosis in paucibacillary sputum: performances of the Xpert MTB/RIF ultra compared to the Xpert MTB/RIF, and IS6110 PCR. Diagnostic microbiology and infectious disease. 2019;94(4):365-70. Epub 2019/04/22. doi: 10.1016/j.diagmicrobio.2019.02.008.

14. World health organization. Lateral flow urine lipoarabinomannan assay (LF-LAM) for the diagnosis of active tuberculosis in people living with HIV. Policy update (2019). Geneva, Sweazerland WHO; 2019.

15. Huerga H, Ferlazzo G, Bevilacqua P, Kirubi B, Ardizzoni E, Wanjala S, et al. Incremental Yield of Including Determine-TB LAM Assay in Diagnostic Algorithms for Hospitalized and Ambulatory HIVPositive Patients in Kenya. PLoS ONE 2017;12(1).

16. Lawn SD, Kerkhoff AD, Burton R, Schutz C, Boulle A, Vogt M, et al. Diagnostic accuracy, incremental yield and prognostic value of Determine TB-LAM for routine diagnostic testing for tuberculosis in HIV-infected patients requiring acute hospital admission in South Africa: a prospective cohort. BMC medicine. 2017;15(1):67. Epub 2017/03/23. doi: 10.1186/s12916-017-0822-8.

17. Peter JG, Zijenah LS, Chanda D, Clowes P, Lesosky M, Gina P, et al. Effect on mortality of point-ofcare, urine-based lipoarabinomannan testing to guide tuberculosis treatment initiation in HIV-positive hospital inpatients: a pragmatic, parallel-group, multicountry, open-label, randomised controlled trial. 
Lancet (London, England). 2016;387(10024):1187-97. Epub 2016/03/14. doi: 10.1016/s01406736(15)01092-2.

18. Bjerrum S, Schiller I, Dendukuri N, Kohli M, Nathavitharana RR, Zwerling AA, et al. Lateral flow urine lipoarabinomannan assay for detecting active tuberculosis in people living with HIV. The Cochrane database of systematic reviews. 2019;10(10):Cd011420. Epub 2019/10/22. doi:

10.1002/14651858.CD011420.pub3.

19. Jimma University. New Medical Center Inaugurated at Jimma University Jimma Jimma University; 2018 [cited 2020 4]. Available from: https://ju.edu.et/.

20. Federal Democratic Republic of Ethiopia Ministry of Health. Guidelines for management of TB, DRTB and leprosy in Ethiopia. Addis Ababa, Ethiopia: Ministry of Health; 2018.

21. Kebede W, Abebe G, Gudina EK, De Vos E, Riviere E, Van Rie A. Role of empiric treatment in hospitalized patients with Xpert MTB/RIF-negative presumptive pulmonary tuberculosis: A prospective cohort study. International Journal of Infectious Diseases. 2020;97:30-7. doi: https://doi.org/10.1016/j.ijid.2020.06.011.

22. World health organization. Improving the diagnosis and treatment of smear-negative pulmonary and extra-pulmonary tuberculosis among adults and adolescents. Geneva, Sweazerliand: WHO; 2007.

23. The Global Laboratory Initiative (GLI). Mycobacteriology Laboratory Manual. gli: Stop TB Partnership; 2014.

24. Cepheid. Xpert MTB/RIF. Solan, Sweden, Cepheied 2012.

25. Zifodya JS, Kreniske JS, Schiller I, Kohli M, Dendukuri N, Schumacher SG, et al. Xpert Ultra versus Xpert MTB/RIF for pulmonary tuberculosis and rifampicin resistance in adults with presumptive pulmonary tuberculosis. The Cochrane database of systematic reviews. 2021;2:Cd009593. Epub 2021/02/23. doi: 10.1002/14651858.CD009593.pub5.

26. Esmail A, Pooran A, Sabur NF, Fadul M, Brar MS, Oelofse S, et al. An Optimal Diagnostic Strategy for Tuberculosis in Hospitalized HIV-Infected Patients Using GeneXpert MTB/RIF and Alere Determine TB LAM Ag. Journal of clinical microbiology. 2020;58(10). Epub 2020/07/31. doi: 10.1128/jcm.0103220.

27. Huerga H, Ferlazzo G, Wanjala S, Bastard M, Bevilacqua P, Ardizzoni E, et al. Mortality in the first six months among HIV-positive and HIV-negative patients empirically treated for tuberculosis. BMC infectious diseases. 2019;19(1):132. Epub 2019/02/13. doi: 10.1186/s12879-019-3775-Z.

\section{Figures}


250 Xpert MTB/RIF negative inpatients enrolled

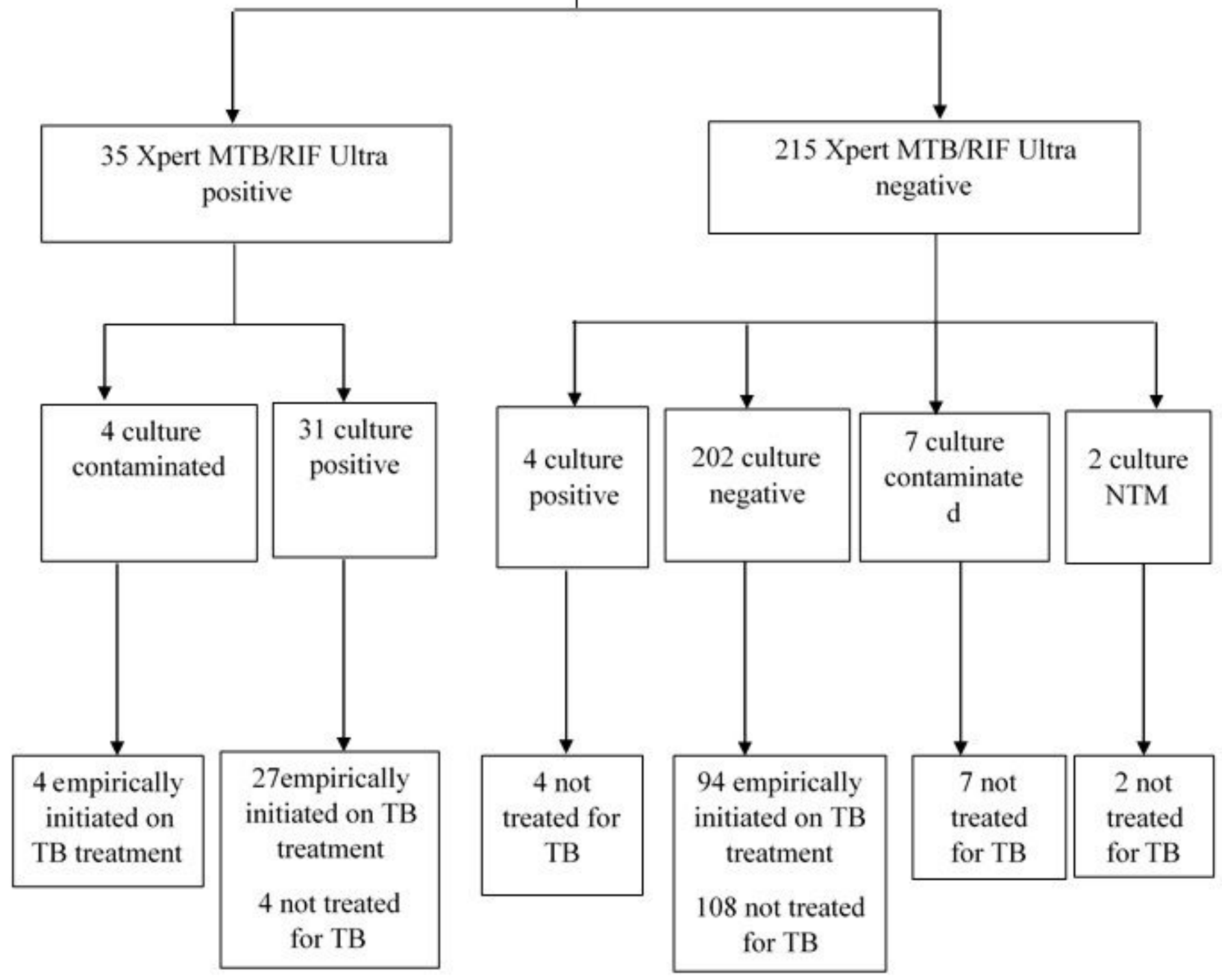

\section{Figure 1}

Patient enrollment flowchart showing the number of patients enrolled, Xpert Ultra result, culture result and treatment status distribution. Abbreviation: TB, tuberculosis; MTB, Mycobacterium tuberculosis; NTM, non-tuberculosis mycobacteria. 


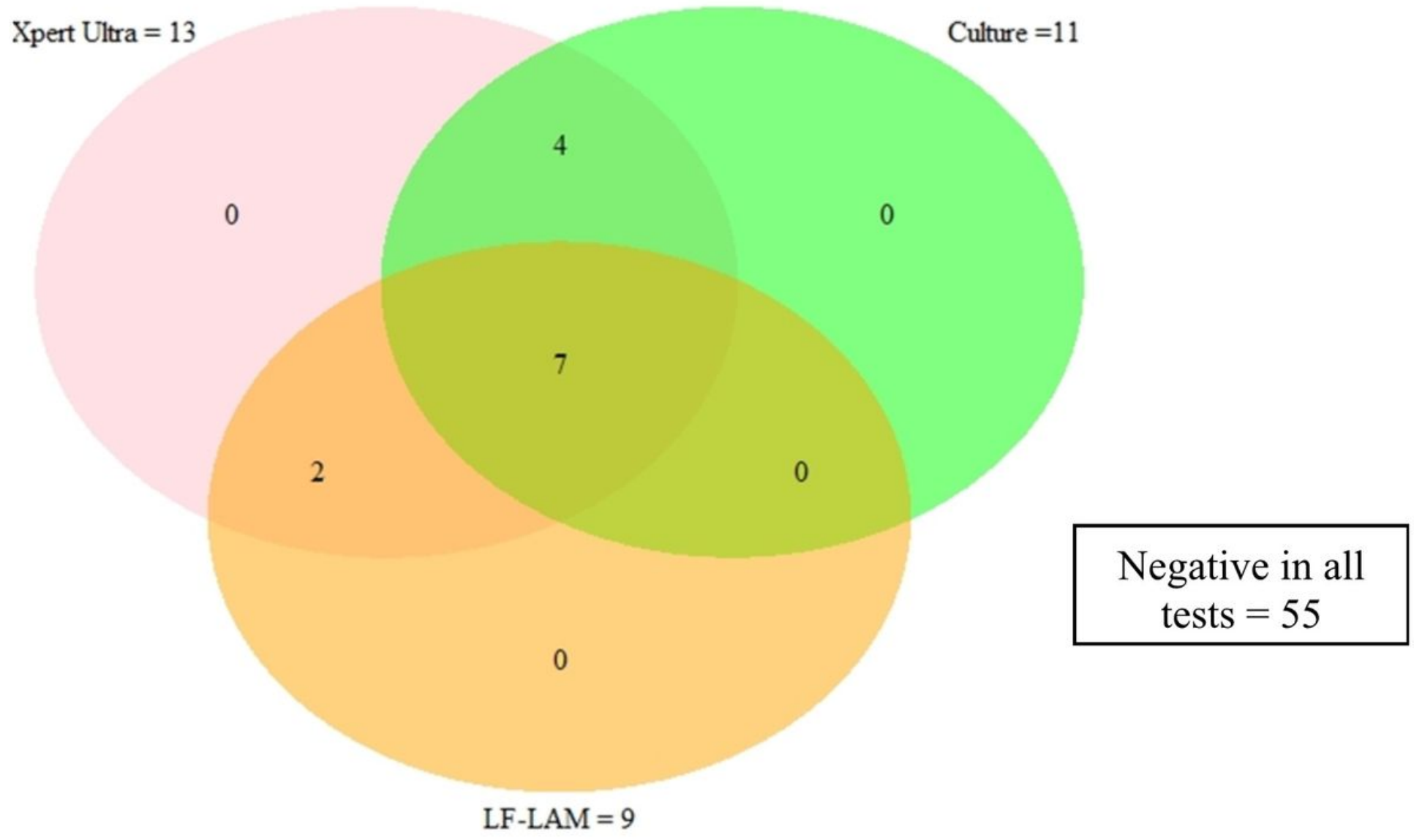

\section{Figure 2}

Venn diagram showing bacteriological confirmation using sputum culture, Xpert Ultra and LF-LAM assay on urine specimen from 52 HIV-positive and 16 severely ill HIV negative Xpert MTB/RIF-negative hospitalized patients with presumptive pulmonary TB enrolled from Jimma University Medical Center, Oromia, Ethiopia, December 2018 to June 2019 ( $\mathrm{N}=68)$. Abbreviation: Xpert Ultra, Xpert MTB/RIF Ultra; LF-LAM, Lateral Flow Urine Lipoarabinomannan; HIV, Human Immunodeficiency virus.

\section{Supplementary Files}

This is a list of supplementary files associated with this preprint. Click to download.

- Supplementaryfile.docx 